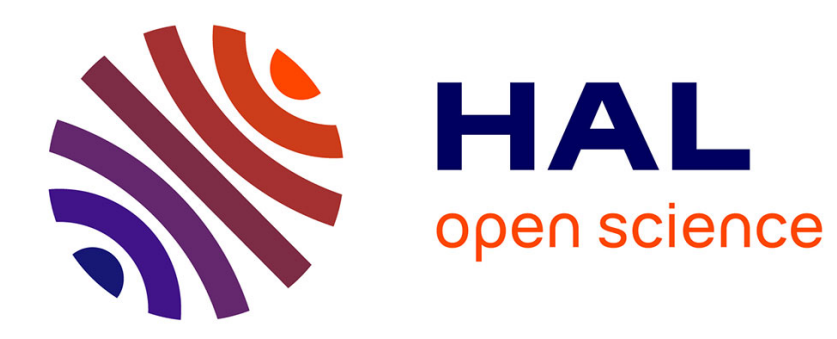

\title{
Planarization and edge bead reduction of spin-coated polydimethylsiloxane
}

\author{
Tiffany Baëtens, S. Arscott
}

\section{To cite this version:}

Tiffany Baëtens, S. Arscott. Planarization and edge bead reduction of spin-coated polydimethylsiloxane. Journal of Micromechanics and Microengineering, 2019, 29 (11), pp.115005. 10.1088/13616439/ab3b18 . hal-02345333

\section{HAL Id: hal-02345333 \\ https://hal.science/hal-02345333}

Submitted on 6 Jan 2021

HAL is a multi-disciplinary open access archive for the deposit and dissemination of scientific research documents, whether they are published or not. The documents may come from teaching and research institutions in France or abroad, or from public or private research centers.
L'archive ouverte pluridisciplinaire HAL, est destinée au dépôt et à la diffusion de documents scientifiques de niveau recherche, publiés ou non, émanant des établissements d'enseignement et de recherche français ou étrangers, des laboratoires publics ou privés. 


\title{
Planarization and edge bead reduction of spin-coated polydimethylsiloxane
}

\author{
Tiffany Baëtens and Steve Arscott
}

Institut d'Electronique, de Microélectronique et de Nanotechnologie (IEMN), CNRS, The University of Lille, Cité Scientifique, 59652 Villeneuve d'Ascq, France.

E-mail: steve.arscott@univ-lille.fr

\begin{abstract}
Films of polydimethylsiloxane (PDMS) can be obtained by spin coating for a range of applications in microengineering and microsystems. However, the spin coating of PDMS films in the 100-1000 $\mu \mathrm{m}$ thickness range invariably results in the formation of non-negligible 'edge beads'. If not removed, such features could cause problems if the PDMS films were to form part of a multi-step planar process involving e.g. contact photolithography. Here, we study the reflow planarization and edge bead reduction of spin-coated PDMS films obtained in the spin-speed range (250-500 rpm). We provide an optimized technical solution for the planarization and associated edge bead removal-along with useful spin speed versus thickness data of PDMS films. Our approach takes into consideration the reflow planarization time and the polymerization time. We demonstrate the usefulness of our method by conducting photolithography on the planarized PDMS films by using a commercial spin-coated photoresist $\left(A Z^{\circledR} 1518\right)$ - clearly showing that the photolithographic resolution is improved for planarized PDMS films compared to non-planarized PDMS films. Finally, the findings suggest that if a planarized PDMS film having a thickness of $\sim 1000 \mu \mathrm{m}$ is required, a single very low spin speed coating followed by an enhanced reflow planarization is preferable to multiple higher spin speed coatings and planarization
\end{abstract}


steps. The findings should be useful to those working to develop stretchable technologies using planar means who wish to integrate PDMS films into more complex systems.

Keywords: polydimethylsiloxane, PDMS, thin film, spin coating, edge bead, reflow planarization, polymerization, photolithography, planar processes

\section{Introduction}

Polydimethylsiloxane (PDMS) [1-3] is a common polymeric material frequently employed in microengineering and microsystems. These uses range from established technologies e.g. moulding microchannels [4-7] and soft lithography [8,9] to emerging technologies such as flexible $[10,11]$ and stretchable systems [12-17]. In the latter context, where more system complexity may be required, integration of PDMS into standard planar processes (involving lithography, deposition, and etching) would open up the way to the development of original processing routes for the fabrication of more complex systems. Such approaches will require the film thickness and planarity (flatness) to be carefully controlled during the processing. Spin coating of pre-cured PDMS liquid mixture enables thickness control-resulting thin films (thickness range 1-100 $\mu \mathrm{m}$ ) that have good planarity [18-22]. However, the spin coating of thicker films (thickness range 100-1000 $\mu \mathrm{m}$ ), potentially required to serve as substrate material for flexible and stretchable systems, result in non-negligible 'edge beads' at the periphery of the wafer [20]. Large edge beads are difficult to remove using standards techniques such as spray removal. They can be removed mechanically [23] - but scalability is a potential issue in this approach. Removal of edge bead via 'cutting' post-cured material if often resorted to in the prototyping of proofof-concept systems, but this can result in film delamination and ejected particulate matter which can 
prove to be difficult to remove from the film surface. In addition, planar approaches do not fare well with such manual interventions, e.g. films cracking can occur due to mechanical stresses and strains. Alternatively, it is well known that reflow planarization [24-29] can be used to remove-or at least reduce-edge beads in spin-coated films. Reflow planarization involves the harnessing of the fluid properties of the pre-cured spin-coated liquid to cause a reflow of the liquid film which results in an even film surface prior to-or indeed during-film curing. We study here the reflow planarization of spincoated commercial PDMS (Sylgard ${ }^{\mathrm{TM}}$ 184) and the subsequent photolithography of a common photoresist on the now solid PDMS surface. Our aim is to produce useful guidelines and results for the process engineer and researcher working in the field of soft planar technologies involving such PDMS films. We show how an appropriate planarization process can be used to effectively reduce edge beads and produce a high-planarity solid PDMS film which can be used as a stretchable host substrate. In addition, we also show how such planarized PDMS films can be spin coated multiple times in order to achieve level films in the upper 100-1000 $\mu$ m range. Finally, we show how such films can be processed using standard photolithographic techniques to achieve a photolithographic resolution of $\sim 2 \mu \mathrm{m}-$ much better than on non-planarized PDMS films containing edge beads and non-planar surfaces.

\section{Experimental}

\subsection{Materials used in the study}

Double-sided polished 3 inch $(7.62 \mathrm{~cm})$ diameter 100 orientated silicon wafers (Siltronix, France) were used for the spin coating of the PDMS films. A commercial PDMS elastomer kit Sylgard ${ }^{\mathrm{TM}} 184$ (Dow Corning, USA) was used to prepare the PDMS base/curing agent mixtures. The photolithography was conducted using a commercial positive photoresist $\left(A Z^{\circledR} 1518\right)$ purchased from MicroChemicals $\mathrm{GmbH}$, Germany, and developed using $A Z^{\circledR} 400$ (Microchemicals GmbH, Germany). 


\subsection{Instruments and techniques used in the study}

A commercial spin coater Delta 10 BM (Suss MicroTec Lithography GmbH, Germany) was used to coat the silicon wafers with the PDMS mixture. An in-house fabricated chromium/quartz photolithographic mask containing features sizes down to $2 \mu \mathrm{m}$ was fabricated at the laboratory. The PDMS films were cured on a levelled $\left( \pm 0.1^{\circ}\right)$ commercial precision hot plate $\left( \pm 0.5^{\circ} \mathrm{C}\right)$ (Prazitherm, Germany). The photolithography was performed using a commercial mask aligner MA6/BA6 240-400 nm (Suss MicroTec Lithography $\mathrm{GmbH}$, Germany). Surface profiling was performed using a commercial precision surface profiler DektakXT (Bruker, USA). The surface profile and thickness data of all the silicon wafers used in the study was gathered prior to PDMS spin coating. Film and wafer thicknesses were measured using a commercial thickness gauge/comparator (Mitutoyo, Japan)-accurate to within $\pm 1 \mu \mathrm{m}$. For the latter measurement it is important to use a small piece of silicon (area $\sim 25 \mathrm{~mm}^{2}$ ) to avoid potential errors in the measured thickness due the probe of the thickness gauge deforming the PDMS. For the photolithography tests, the photoresist was pre-exposure baked in a commercial cleanroom box furnace (Memmert GmbH, Germany). The samples were visually inspected using a VHX-5000 digital microscope (Keyence, Japan). Finally, it is important to describe how the surface profiling data was analysed. The commercial surface profiler has a maximum range of $50 \mathrm{~mm}$ - the data was gathered by setting zero to be at the wafer edge. The surface profile data was gathered using a $50 \mathrm{~mm}$ range passing through the centre of the wafer-which is positioned at $38.1 \mathrm{~mm}$ from the wafer edge. In order to level the surface profile data, in each case two points were used on each data set which were equally spaced from the wafer centre and positioned at $5 \mathrm{~mm}$ from the wafer centre-these were the points used for the automatic data levelling. As PDMS is a soft material, a force equal to $0.1 \mathrm{mg}$ was employed for the 
surface profiling. Each surface profile scan resulted in $\sim 36,000$ points-giving an in-plane length resolution of $\sim 1.4 \mu \mathrm{m}$.

\subsection{Fabrication of the samples}

All the fabrication processes and measurements were performed in an ISO 5/7 cleanroom. The base and curing agent of the commercial PDMS kit were mixed in 10:1 (w:w) proportions to produce sufficient volume to coat several 3 inch silicon wafers ( 20 wafers were used for the experiments). Following mixing, the liquid PDMS mixture was de-gassed by placing the mixture in a chamber pumped using a rotary pump (20 minutes). For the spin coating, the silicon wafers were used directly from the supplier's box-they had received an 'RCA clean' [30] at the manufacturers. The wafers did not undergo a surface treatment before spin coating-thus the surface is a native oxide. A nitrogen gun was used at the last moment to remove any particulate matter from the wafer surface prior to coating with the PDMS mixture.

Figure 1 shows a schematic diagram illustrating the different steps of the fabrication process developed here to produce the planarized films of PDMS. 


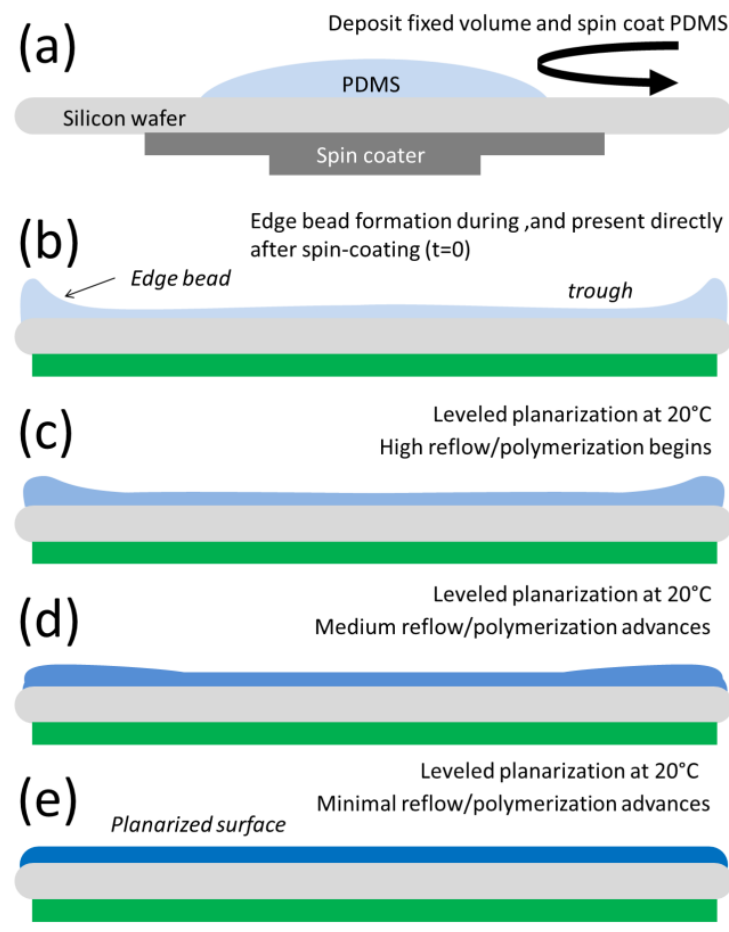

(f) $100-1000 \mu \mathrm{m} \quad$ Near-zero reflow/near-complete polymerization $\downarrow$ $\uparrow$

(g)

Curing at $100^{\circ} \mathrm{C}(0.5 \mathrm{~h})$ Zero-edge bead complete polymerization-high viscosity

Figure 1 The fabrication process for the planarization of spin-coated PDMS films. (a) A fixed volume (8 $\mathrm{ml}$ ) of PDMS base/curing agent mix is deposited onto the centre of the silicon wafer followed by a spin coating step. (b) The PDMS film having large edge beads immediately after spin-coating. (c-f) The sample undergoing reflow planarization. (g) The sample is cured on a levelled hot-plate (gold) and finally transferred to a box furnace. The different colours (light blue to violet) of the PDMS film indicate the different stages of polymerization. 
The following passage describes the planarization process illustrated in Figure 1. A fixed volume $(8 \mathrm{ml})$ of PDMS base/curing agent mixture was poured onto the centre of the silicon wafer-immediate wetting and spreading of the mixture leads to a PDMS 'puddle' having a diameter of $\sim 4 \mathrm{~cm}$ in the centre of the silicon wafer [see Figure 1(a]. The PDMS mixture was spin coated onto the silicon wafers at speeds of $250 \mathrm{rpm}, 350 \mathrm{rpm}$, and $500 \mathrm{rpm}$ (a spin coating acceleration of $350 \mathrm{rpm} \mathrm{s}^{-1}$ ) for 30 seconds. An edge bead and an uneven PDMS surface is visible directly following spin coating [see Figure 1(b)]. Once the spin coating had finished, the wafers were carefully placed onto levelled copper blocks having a diameter of approximately $4 \mathrm{~cm}$-this eliminates problems caused by any PDMS mixture present on the bottom of the wafer in proximity to the wafer edges [see Figure 1(b)]. The blocks, onto which the wafers were placed, had been carefully pre-levelled to a precision $\pm 0.1^{\circ}$-we emphasize that this is a critical point for long time planarization of such films. The accuracy $\left(<0.1^{\circ}\right)$ of the spirit level was checked with a calibrated levelled surface. A non-sealed plastic screen was placed over the samples and they were left in this state $\left(20^{\circ} \mathrm{C}\right)$ for a number of hours $(1-24$ hours).

During this planarization period-see Figures 1(b-f)-two main effects occur: (i) reflow of the PDMS mixture causing planarization and edge bead reduction and (ii) polymerization of the PDMS mixture which increases the viscosity of the PDMS. Note that the increase in viscosity leads to a reduction in the reflow which effectively decelerates the reflow planarization-these are competing processes. The reflow planarization occurs in the initial period when the viscosity of the PDMS is still relatively low. When the polymerization has sufficiently advanced to enable handling of the sample (i.e. tweezers do not leave defects in the PDMS film) - the sample is transferred to a box furnace-see Figure 1(g). The samples were cured in the commercial box furnace at a temperature of $100^{\circ} \mathrm{C}$ for 20 minutes according to the suppliers data sheet-as the viscosity of the cured PDMS is now high, it not necessary to have a perfectly levelled box furnace shelf. In order to test the possibility of multi-layer deposition and planarization, one of the samples (previously spin coated at $250 \mathrm{rpm}$ and planarized for 24 hours) had an 
extra PDMS spin coating ( $8 \mathrm{ml}$ of PDMS mixture/ $350 \mathrm{rpm}$ for $20 \mathrm{~s}$ ). The spin coated PDMS films were planarized for 0 hours, 5 hours, 24 hours, and 72 hours to study the effect of planarization time on the final planarity of the film. Some 'non-planarized' PDMS films were placed in the box furnace directly after spin-coating-see Supplementary Information for a schematic diagram of this process.

Finally, following the surface profiling measurements, some of the wafers were spin coated with the commercial photoresist, exposed to UV radiation $(365 \mathrm{~nm})$ via the photomask, and developed in the commercial developer solution (see Section 2). The thickness and planarity of the silicon wafers used was carefully measured using the thickness gauge and the surface profiler. The planarity was found to be $<3$ $\mu \mathrm{m}$ and the thickness variation $+/-10 \mu \mathrm{m}$. For completeness, the bevelling at the silicon wafer edge was inspected using the optical microscope-as it is known that the specific profile of an edge has an effect of the spreading of a liquid [31] - see Supplementary Information.

\section{Results and discussion}

\subsection{Coating thickness versus spin speed for PDMS on silicon}

Figure 2 shows the measured thickness of spin-coated PDMS on silicon wafers. Two types of PDMS films were fabricated: termed 'non-planarized' and 'planarized' (see Section 2). The spin speed was varied from $250 \mathrm{rpm}$ to $5000 \mathrm{rpm}$. 

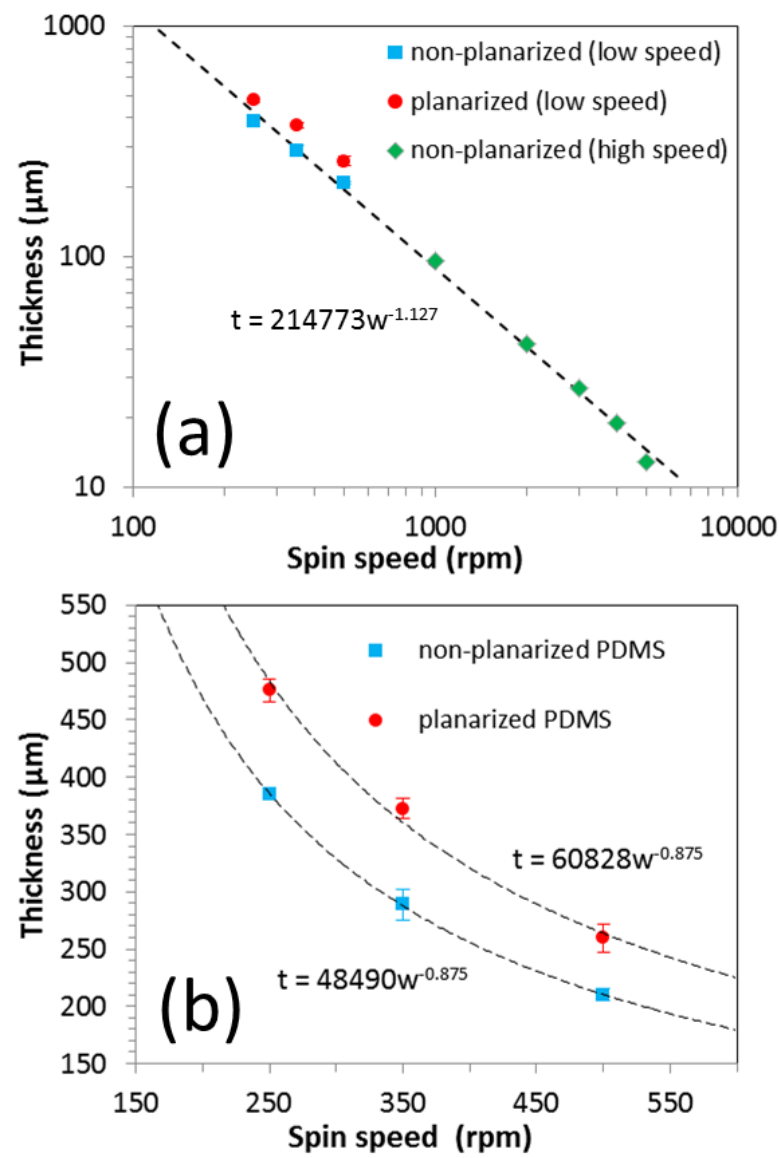

Figure 2. Spin coating of PDMS films on silicon wafers. (a) Measured PDMS film thickness over the spin speeds range $250 \mathrm{rpm}$ to $4000 \mathrm{rpm}$. (b) The low spin-speed range $250-500 \mathrm{rpm}$. The non-planarized PDMS films are indicated by filled light blue squares - the planarized PDMS films are indicated by filled red circles (at low spin speed) and filled green diamonds (at high spin speeds 1000-4000 rpm). The dashed black lines correspond to power law fits [32] to the data-the equations are given. The spin coating acceleration is $350 \mathrm{rpm} \mathrm{s}^{-1}$, the spin coating time is $30 \mathrm{~s}$, and the curing temperature/time is $100^{\circ} \mathrm{C} / 20$ minutes.

Figure 2(a) shows the measured thickness of spin coated PDMS over the whole spin speed range. If we consider the non-planarized films, the experimental data agrees well with a reciprocal curve fitting. 
Previous studies concerning the spin coating of PDMS also found this relationship $[19,20]$. Let us now focus on the low spin-speed data (250-500 rpm) which is shown in Figure 2(b), and compare the planarized and non-planarized PDMS films. The planarized PDMS films are always thicker than the nonplanarized films-with the difference in thickness increasing slightly as the spin speed is decreased from $500 \mathrm{rpm}$ to $250 \mathrm{rpm}$. The two sets of restricted data can be accurately fitted with power laws (should dashed curves) which are given in Figure 2(b) - these should be of practical use for a processing engineer. Concerning the curve fitting given, for non-planarized PDMS films (Sylgard 184) having a thickness $<100 \mu \mathrm{m}$, Schneider et al [19] and Koschwanez et al [20] found a near reciprocal relationship with spin speed. Finally, we also used a double coating using two spin speeds and two planarization steps of 24 hours each. By spin coating at $250 \mathrm{rpm}$ and recoating at $350 \mathrm{rpm}$ after planarization and curingwe were able to produce a planarized PDMS film thickness of $1194.3 \mu \mathrm{m}$ (not plotted on Figure 2). This is greater than the expected thickness $(\sim 850 \mu \mathrm{m})$ of the two combined coatings and planarizations. However, Koschwanez et al [20] found that a thicker PDMS film is obtained when spin coating PDMS mixture onto PDMS compared to glass. The findings can also be compared to those of Mata et al [2]. They studied the spin coating of PDMS (10/1 - Sylgard 184) at low spin speeds 50-1000 rpm onto oxidized silicon wafers. They reported a thickness variation between $1085 \mu \mathrm{m}$ and $55 \mu \mathrm{m}$ as the spin speed is increased. They noted the importance of levelling during curing, as is stressed here, and the specific wafer surface. However, they apparently did not employ reflow planarization to the PDMS films - this could explain our larger film thicknesses observed here. It should also be noted that the air flow influence the final thickness [33] - the spin coating here was performed without closing the cover which, in principle, leads to thinner films. Let us now use surface proofing to investigate the differences in the planarized and non-planarized PDMS films as a function of spin coating speed. 


\subsection{Surface profiling of edge bead features and for spin-coated PDMS on silicon}

Figure 3 shows the results of the surface profiling of non-planarized and planarized spin-coated PDMS films on silicon wafers as a function of spin speed. All 'planarized' PDMS films were left to reflow for 24 hours. Note that '0 hours' condition corresponds to the PDMS sample being transferred directly to a levelled box furnace shelf $\left(100^{\circ} \mathrm{C}\right)$ from the spin coater. The viscosity of the PDMS will be initially reduced in this case due to the rapid change of temperature [34] thus initially enhancing reflow [35] compared to room temperature planarization and curing. However, the increased temperature also enhances the polymerization rate substantially, rapidly 'fixing' the edge-bead profile into what we measure here-and refer to-as the ' 0 hours' state of planarization. 

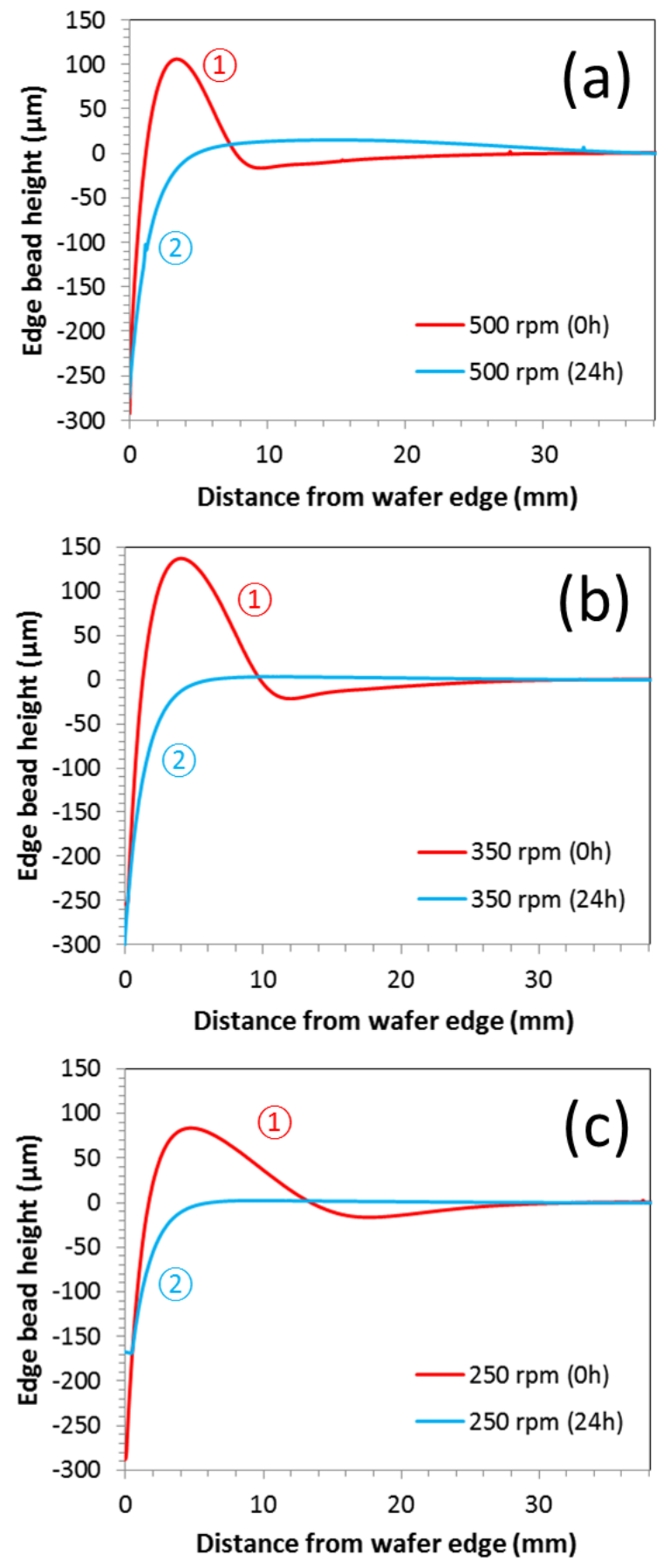

Figure 3. The surface profile of the PDMS films before and after planarization for various spin-speeds. The PDMS films are spin coated onto silicon wafers at (a) $500 \mathrm{rpm}$, (b) $350 \mathrm{rpm}$, and (c) $250 \mathrm{rpm}$. For the planarized films, the planarization time is 24 hours for all planarized samples. The non-planarized curves 
are indicated red and labelled '(1)'. The planarized curves are indicated light blue and labelled '(2)'. The abscissae correspond to distance from the silicon wafer edge-half the wafer was profiled.

There are several observations which can be made from Figure 3. First, all non-planarized films result is a relatively large edge bead compared to the PDMS thickness (cf. Figure 2)-this is seen by looking at the red curves labelled (1) in the figure. This edge bead has an associated 'trough' feature which is positioned towards the centre of the wafer. The edge bead height is larger than the trough depth for all three spin speeds. The total length of the edge bead plus trough feature is observed to increases from approximately $20 \mathrm{~mm}$ to $30 \mathrm{~mm}$ as the spin speed is reduced from $500 \mathrm{rpm}$ to $250 \mathrm{rpm}$. Following planarization for 24 hours, the initial edge bead plus trough feature become smoother. However, better planarization is achieved for PDMS films obtained at lower spin speeds - this is seen by comparing the light blue (2) curves in the figure. This explains the data given in Figure 2-the final planarized PDMS thicknesses (filled red circles) are larger than the non-planarized thicknesses (filled blue squares) due to the reflow of material towards the centre of the wafer.

Figure 4 shows extracted data from the surface profiling shown in Figure 2. A guide to where these dimensions are taken are given in Supplementary Figure S3 in the Supplementary Information. First, the edge bead peak height (relative to the PDMS film thickness at the centre of the wafer) shows a maximum-see filled red circles in Figure 4(a). This can be explained via the ejection of matter from the edges at higher spin speeds [36]. At lower spin speeds, little matter is ejected-increasing the spin speed leads to matter accumulating at the wafer edge. Increasing the spin speed still further leads to more ejected matter from the edge resulting in a reduced edge bead. The edge bead position reduces approximately linearly with increasing spin speed-filled blue triangles in Figure 4(a). In contrast to the 
edge bead peak height, the trough depth shows a minimum as the spin speed is increased-and the trough position reduces with increasing spin speed.
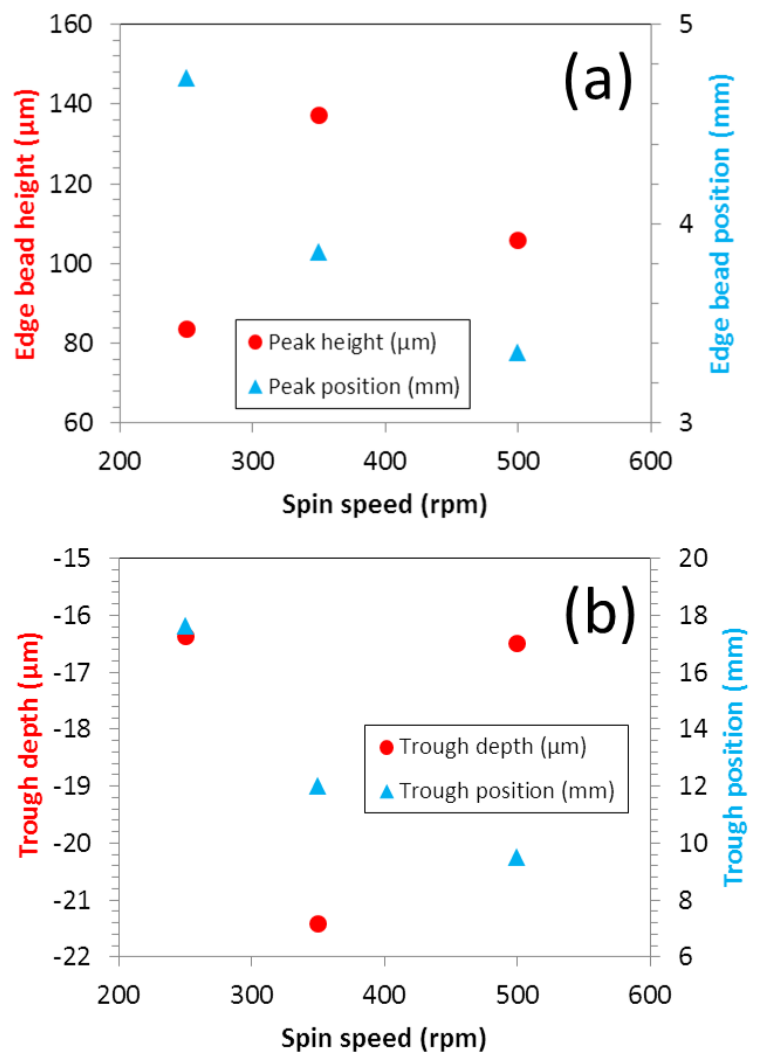

Figure 4. Plotted data from the surface profiling of the non-planarized PDMS films spin coated onto silicon wafers. (a) Maximum edge bead height and position (from the edge of the wafer) versus spin speed. (b) Maximum trough depth and trough position versus spin speed.

Having established that a relatively large edge bead is present for directly-cured $\left(100^{\circ} \mathrm{C}\right)$, spincoated PDMS (0 hours), let us now focus on the planarization and study the effects of planarization time and PDMS thickness on the resulting planarization. Figure 5 shows various surface profiles following 
planarization of spin coated PDMS films for different planarization times ranging from 0 hours to 72 hours-note again that the ' 0 hours' condition corresponds to the sample being transferred directly to the calibrated levelled box furnace shelf from the spin coater.
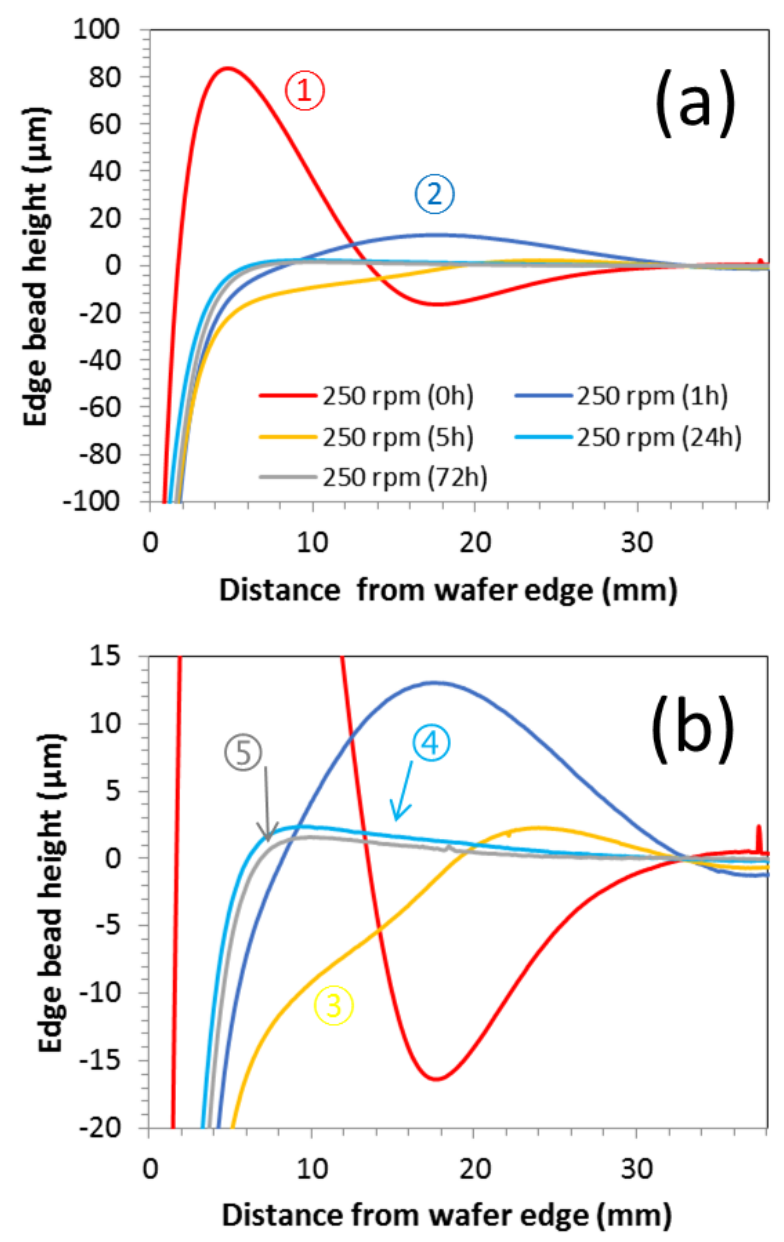

Figure 5. The surface profile of spin-coated PDMS for different planarization times. (a) Edge bead height versus distance from wafer edge and (b) a zoom of the ordinate. The profiles are given for planarization times of 0 hours (red curves (1)), 1 hour (dark blue curves (2)), 5 hours (yellow curves (3)), 24 hours (light 
blue curves (4)), and 72 hours (grey curves (5)). The spin speed in all cases is $250 \mathrm{rpm}$. The abscissae correspond to distance from the silicon wafer edge.

Again there are several observations which can be made. First, not surprisingly the edge bead height diminishes with increasing planarization time. After 1 hour the edge bead has diminished in size and advanced towards the centre of the wafer-this is predicted in gravity-driven planarization [37]. After 5 hours the reflow is still ongoing - the edge bead position has further advanced towards the wafer centre. After 24 hours the planarization of the PDMS is near complete-the peak height compared to the centre of the wafer is $\sim 2 \mu \mathrm{m}$. After 72 hours the planarization has progressed but only $\sim 0.5 \mu \mathrm{m}$ is gained compared to $2 \mu \mathrm{m}$ at 24 hours. Let us now compare the surface profiles of spin coated PDMS films for different spin speeds.

Figure 6 shows the surface profiles of spin-coated PDMS films for different spin coating speeds (250-500 rpm). 

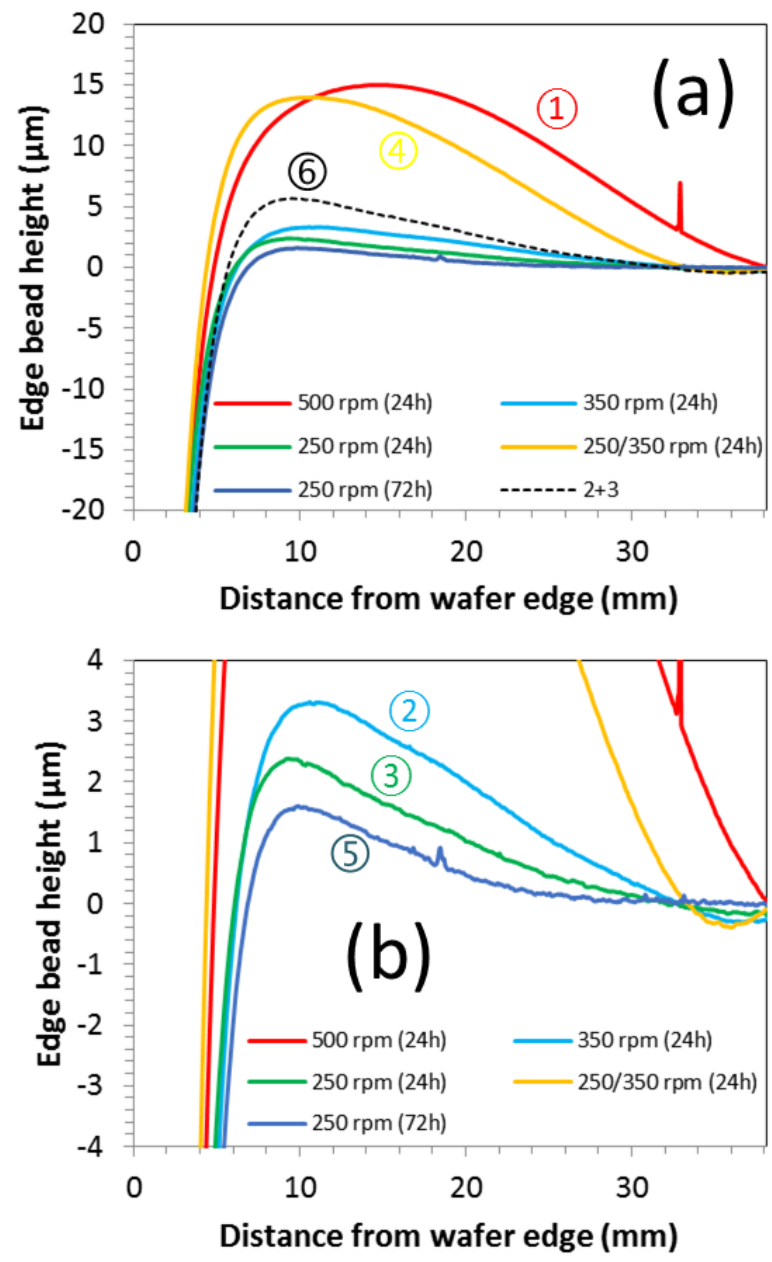

Figure 6. The surface profile of planarized PDMS films for different spin coating speeds. (a) Surface profile height versus length from wafer edge and (b) a zoom of the ordinate. The profiles are given for spin coating speeds of $500 \mathrm{rpm}$ (red curves (1)), $350 \mathrm{rpm}$ (light blue curve (2)), $250 \mathrm{rpm}$ (green curves (3)), $250 \mathrm{rpm}$ followed by $350 \mathrm{rpm}$ (yellow curves (4)), and $250 \mathrm{rpm}$ (for a planarization time of 72 hours) (dark blue curves (5)). The dashed black curve (6) corresponds to the mathematical sum of the $250 \mathrm{rpm}$ (3) and $350 \mathrm{rpm}$ (2) curves. The abscissae correspond to distance from the silicon wafer edge.

For a planarization time of 24 hours the most planar PDMS film is obtained using the lowest spin speed, i.e. the thickest PDMS film. Indeed, there is a clear trend of reducing peak edge bead height as 
the spin speed is reduced. This can be seen by comparing the red (1), light blue (2), and green (3) curves in Figure 6. Note that the spike in the red curve labelled (1) in Figure 6 is a particle on the PDMS surface. If the planarization time is extended to 72 hours (as is the case of the PDMS sin coated at 250 rpm heresee the dark blue curve (5), the edge bead height is still further reduced indicating that reflow is not complete at 24 hours.

Interestingly, if a doubly coated/planarized layer is used to achieve at thickness of $1194.3 \mu \mathrm{m}$ (see above), then a relatively larger edge bead is obtained-see the yellow curve (4) in Figure 6 . As a comparison the black dashed curve '(6) in Figure 6 shows the mathematical sum of the profiles obtained at $250 \mathrm{rpm}$ and $350 \mathrm{rpm}$. The large edge bead that is obtained when the processes are combined suggests the planarization is slowed when a PDMS films is planarizing on a PDMS surface compared to a silicon surface. The thickness dependence on the underlying surface observed by Koschwanez et al [20] could also be a factor.

Figures 7 plots extracted data from Figures 5 and 6. A guide to where these dimensions are measured is given in Supplementary Figure S3 in the Supplementary Information. First, the edge bead peak height reduces non-linearly with increasing planarization time-see filled red circles in Figure 7(a). The edge bead peak position moves towards the centre of the wafer [37] and then back to the edge of the wafer as the planarization time is increased-see filled blue triangles in Figure 7(a). Kheshgi and Scriven [37] also predicted that viscous films can damp periodically rather than monotonically-this may be what we are observing here after a 5 hour planarization period-see yellow curve (3) in Figure 5 . As the spin speed is increased, the peak edge bead height increases-see filled red circles in Figure 7(b). The edge bead position also increases (i.e. a shift towards the centre of the wafer) as the spin speed is increased-see filled blue triangles in Figure 7(b). Figure 7(c) plots the edge bead height and position as a 
function of PDMS thickness (using Figure 2) to give a practically useful figure. The edge bead height diminishes non-linearly as the total PDMS thickness is increased.
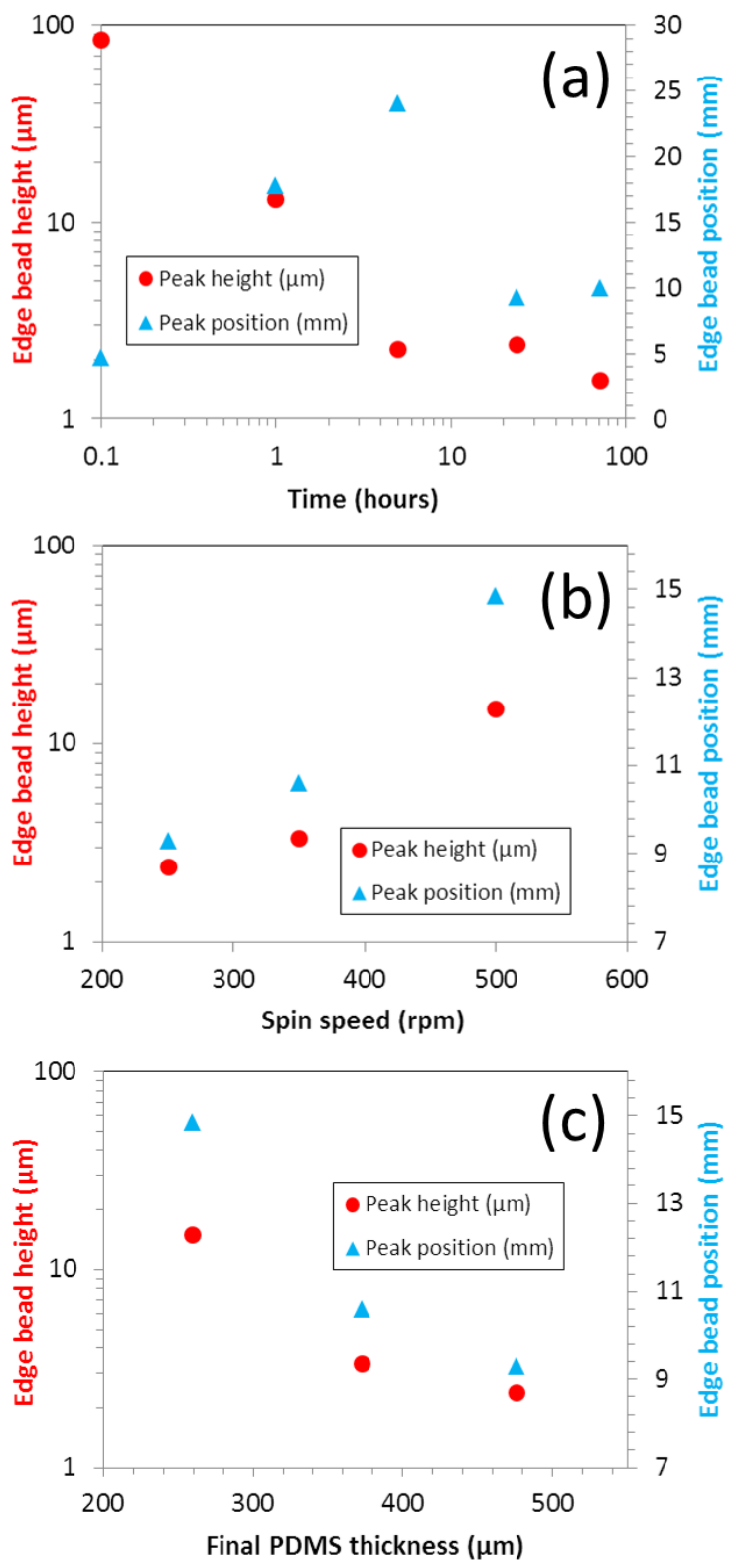
Figure 7. Plotted data from the surface profiling measurements of the planarized PDMS films spin coated onto silicon wafers. (a) Maximum edge bead height and position (from the edge of the wafer) versus planarization time. (b) Maximum edge bead height and position versus spin speed for a planarization time of 24 hours. (c) Maximum edge bead height and position as a function of PDMS thickness.

\subsection{Comparison of the results with a model for planarization}

The modelling of the fluid dynamics of reflow planarization of a viscous liquid film is somewhat involved [37] -even for a liquid having time-invariant physical properties reflowing on a smooth surface [35]. However, the experimental observations of the planarization of the spin-coated PDMS films can be compared to an analytical solution of liquid reflow on a smooth surface [35]. In the case of a gravitydriven reflow, the variation of the height $d$ of a liquid wave-like perturbation having an initial height $d_{0}$ is given by:

$$
d=d_{0} e^{-t / \tau}
$$

where the characteristic reflow relaxation time $\tau$ is given by the following formula:

$$
\tau=\frac{3 \eta \lambda^{2}}{4 \pi^{2} \rho g h^{3}}
$$

$\eta$ and $\rho$ are the viscosity and density of the liquid, $\lambda$ is a wavelength of the perturbation, $g$ is the acceleration due to gravity, $h$ is the average film thickness over the period of the planarization, $d$ is the height of the perturbation at time $t$, and $d_{0}$ is the initial height of the perturbation. The larger the characteristic reflow relaxation time $\tau$, the longer the planarization time. Although this model considers a constant viscosity (which is not the case here-see the discussion below), it allows us to compare the experimental results with the predictive trends of the model. First, the model predicts that thinner PDMS 
films should take longer to planarize for a given initial edge bead thickness-this is observed in the experimental results. The PDMS film thickness range planarized in the study suggests that the characteristic relaxation time can vary considerably $\left(\tau \propto h^{-3}\right)$. This implies that the planarization of the edge beads will be very sensitive to the thickness of the PDMS - this is observed in the experimental results. The model predicts that wider edge beads should take longer to be planarized. In our experimental results, a shorter edge bead $(\sim 25 \mathrm{~mm})$ is associated with a thicker film — see Figure 3(c)— thus both reduce the characteristic reflow relaxation time $\tau$.

Looking at the experimental results we can take $\lambda=25 \mathrm{~mm}, h=430.5, \rho_{P D M S} \sim 970 \mathrm{~kg} \mathrm{~m}^{-3}$. We can use the above equations (1) and (2) to estimate the average viscosity $\eta_{\text {ave }}$ and characteristic time constant $\tau_{\text {ave }}$ of the PDMS which would be required to enable the experimentally observed reduction in the edge bead height (see Figure 5). These average values are plotted below in Figure 8.

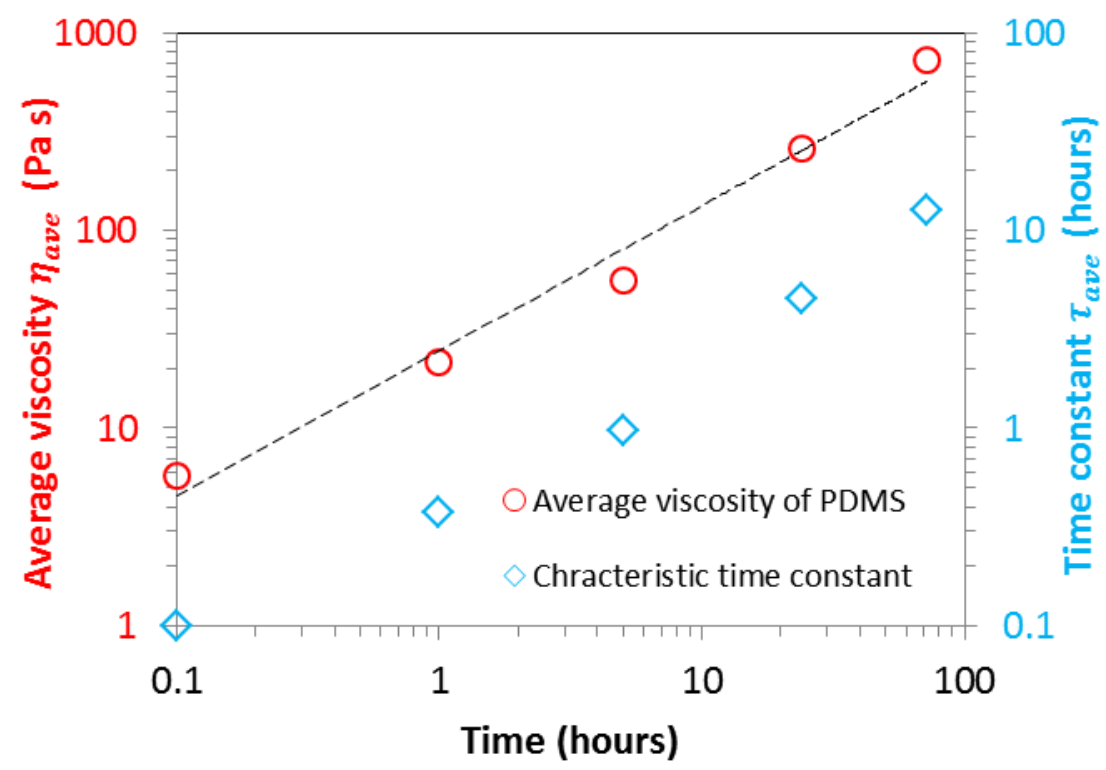

Figure 8. The average viscosity and characteristic time constant of the PDMS base/curing agent mixture obtained from the experimental results of the edge bead reduction. The dashed line corresponds to a power law fit: $\eta_{\text {ave }}=24.64 t^{0.73}$. 
We know from the literature that when curing PDMS the viscosity varies considerably and nonlinearly from $\sim 4 \mathrm{~Pa} s$ to $\sim 1 \times 10^{4} \mathrm{~Pa}$ s from the beginning to the end of the curing. At room temperature the curing time of PDMS is 48 hours [38]. By using the experimental results and the model we are able to estimate an average viscosity for a given planarization period-this is shown in Figure 8. However, we note that to go further in the analysis a more detailed treatment of the problem is necessary. Let us now return to practical issues and demonstrate an advantage of planarized PDMS films using photolithography.

\subsection{Photolithographic resolution on planarized and non-planarized spin-coated PDMS films}

The following section studies the influence of the non-planarity of a PDMS film on the photolithographic resolution of a photoresist deposited onto the PDMS surface. In order to prove the benefits of the planarization technique expounded here we have conducted standard photolithographic steps on the surface of the spin-coated PDMS films. In order to do this a commercial positive photoresist was employed $\left(A Z^{\circledR} 1518\right)$. A patterned standard chromium/quartz photomask-fabricated in our laboratory-containing square holes having side dimensions of $10 \mu \mathrm{m}$ down to $2 \mu \mathrm{m}$ was employed for the ultra-violet photolithographic exposure $(\lambda=365 \mathrm{~nm})$ in a commercial mask aligner. In the practical example here, the thickness of the $A Z^{\circledR} 1518$ photoresist layer is $\sim 1.7 \mu \mathrm{m}$ (measured using a DektakXT surface profiler - Bruker, USA). The masking produced photolithographic 'holes' in the photoresist all over the surface of the PDMS film-importantly right up to the edge of the wafer-in order to experimentally determine the resolution of the photolithography. In practice, in photolithographic 'soft contact' mode-as used here-the pressure of the mask-to-wafer contact is set to 0.2 bar $\left(2 \times 10^{4} \mathrm{~Pa}\right)$. The compression of the PDMS edge bead can thus be estimated if we consider that this pressure acts on 
a PDMS ring having width equal to the edge bead lateral dimension and a thickness equal to the edge bead height. By taking the Young's modulus of the PDMS mix/process used here to be 2 $\mathrm{MPa}$ [3], one can compute that the PDMS is compressed $\sim 5 \mu \mathrm{m}$-this is $\sim 1 \%$ of the total PDMS thickness and is effectively negligible. Figure 9 shows optical micrograph (VHX-5000 digital microscope) of the photomasks used, and the patterning of the photoresist resulting from the photolithography.

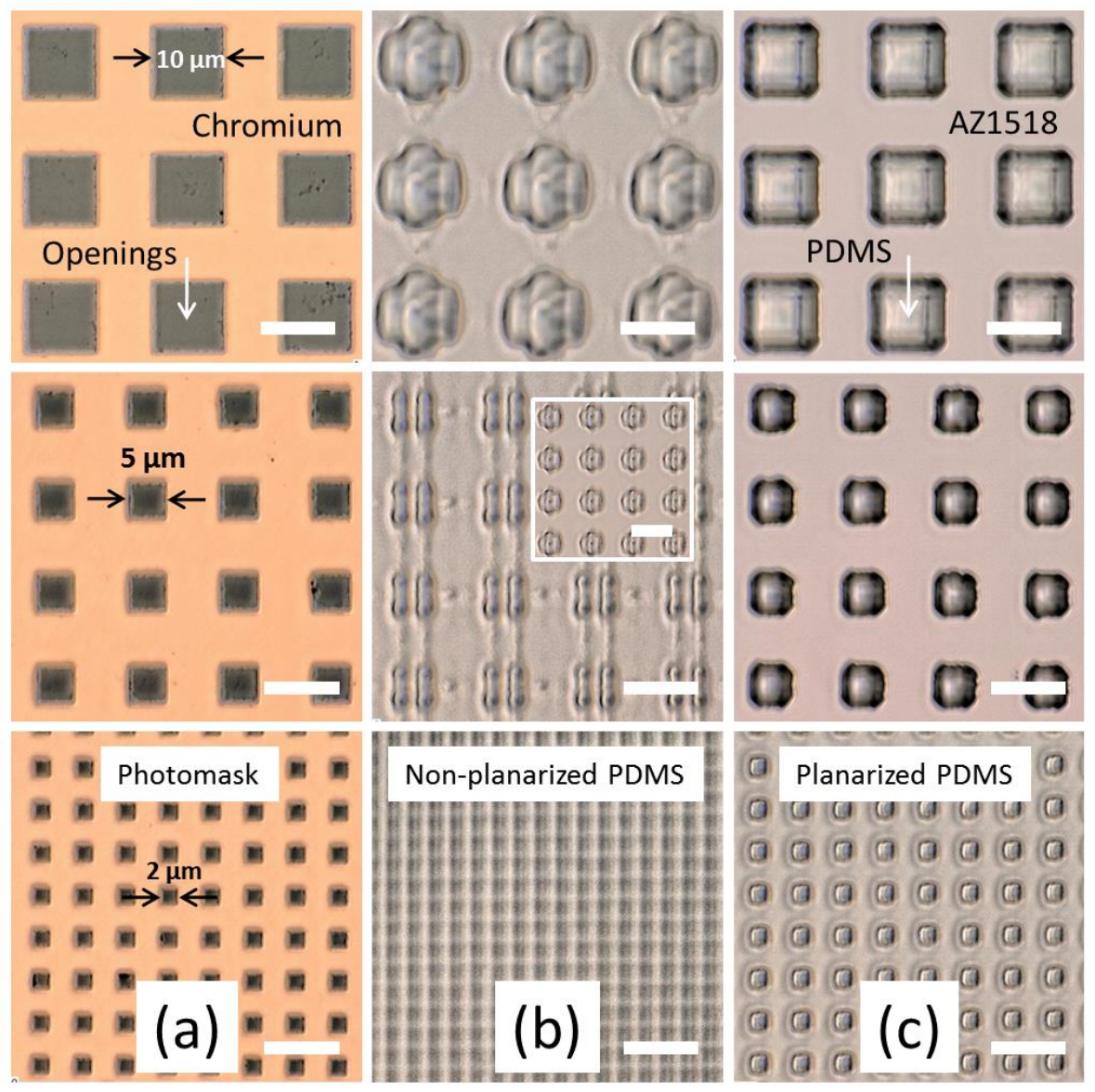

Figure 9. Photolithography of a positive photoresist deposited onto spin-coated PDMS films. (a) Photographs of the chromium/quartz photomask-the mask openings (squares) have a side length of 10 $\mu \mathrm{m}$ (upper image), $5 \mu \mathrm{m}$ (middle image), and $2 \mu \mathrm{m}$ (lower image). (b) Results obtained using the nonplanarized PDMS film. (c) Results obtained using the planarized PDMS film. In (b) and (c) the upper, middle, and lower images correspond to photolithographic openings in the photoresist obtained using 
the corresponding photomask shown in (a). The PDMS films were spin coated at $250 \mathrm{rpm}$. The positive photoresist is AZ1518 (thickness $\sim 1.7 \mu \mathrm{m}$ ). The photographs are taken at $\sim 10 \mathrm{~mm}$ from the edge of the wafer-except the inset to the middle image of (b) which was taken at $\sim 5 \mathrm{~mm}$ from the wafer edge. The photographs were taken using a VHX-5000 digital microscope (Keyence, Japan). All white scale bars indicate $10 \mu \mathrm{m}$.

Figure 9(a) show the size and shape (squares) of the openings in the photomask. Figures 9(b) and 9(c) show the resulting photolithographic patterning of the AZ1518 on non-planarized and planarized spin-coated PDMS. In all cases (except the inset to Figure 9(b)-middle image) the photographs were taken at $10 \mathrm{~mm}$ from the edge of the wafer. For the inset to Figure $9(\mathrm{~b})$, the photograph was taken at 5 $\mathrm{mm}$ from the wafer edge. By comparing Figures $9(\mathrm{~b})$ and $9(\mathrm{c})$ one can see that a photolithographic resolution of $2 \mu \mathrm{m}$ is possible using the photomask at $10 \mathrm{~mm}$ from the wafer edge. In contrast, a poor resolution is obtained using the non-planarized films-except when images are taken at $5 \mathrm{~mm}$ from the wafer edge. In the latter case, this is approximately the position of the peak edge bead height. These results can be understood if the theoretical photolithographic resolution is calculated using the surface profiling data-this is shown in Figure 10. 

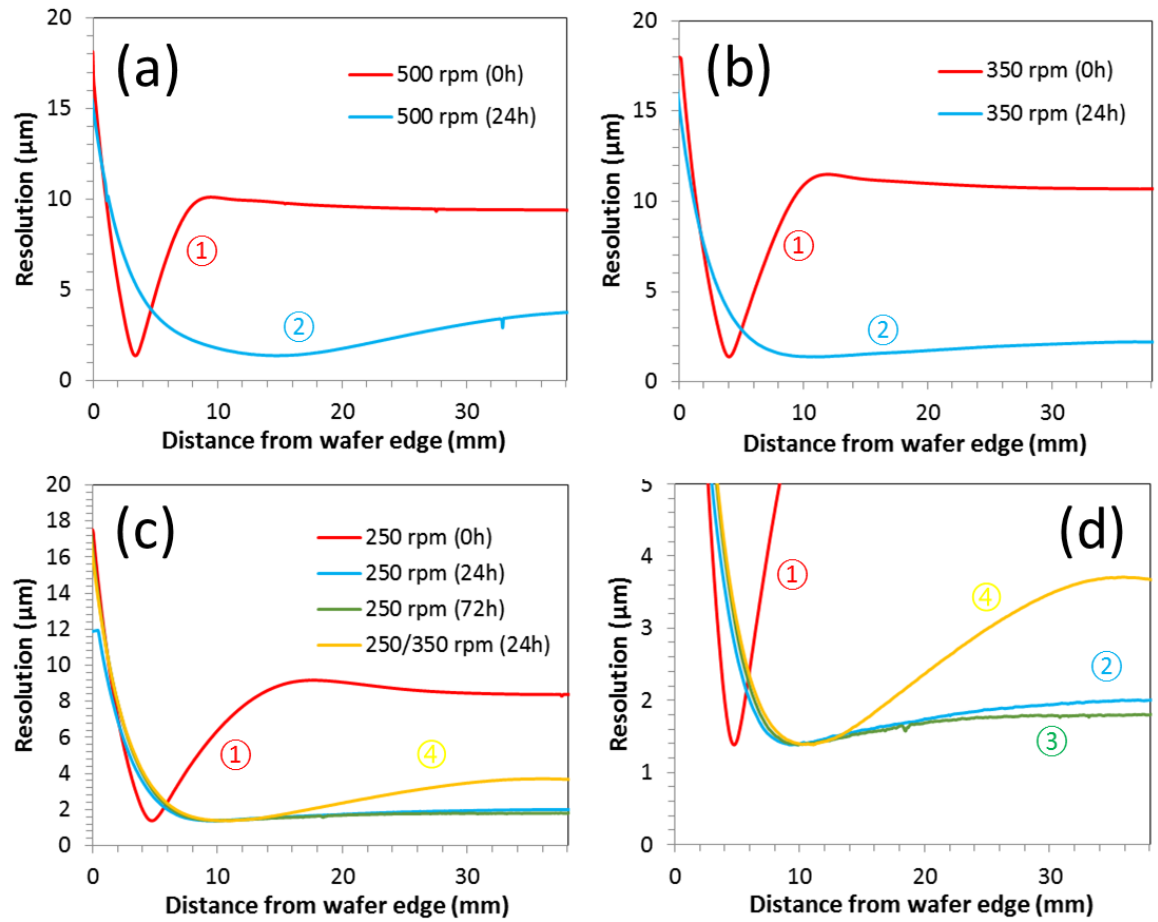

Figure 10. The photolithographic resolution using the non-planarized and planarized PDMS films. The spin coating speeds are (a) $500 \mathrm{rpm}$, (b) $350 \mathrm{rpm}$, and (c) $250 \mathrm{rpm}$. A zoom of the data presented in (c) is shown in (d). The non-planarized results are indicated by the red (1) curves-the planarized data is indicated by the other coloured (2)-(4) curves. The planarization time in all cases is 24 hours.

The resolution $R$ of photolithography is the minimum feature size which is transferable from the mask to the photoresist-it is computed using the following equation [39]:

$$
R=\frac{3}{2} \sqrt{\lambda\left(s+\frac{z}{2}\right)}
$$

Where $\lambda$ is the wavelength of the light used for the exposure, $s$ is the gap distance between the mask and the top of the photoresist layer, and $z$ is the photoresist thickness. In the present work, the gap is due to the presence of the edge bead and the non-uniformity of the cured PDMS film. The 
photolithographic resolution can be computed as a function of lateral distance from the wafer edge from equation (3) above by equating $s$ to the difference between the PDMS surface and the edge bead height. The experimental results above (see Figure 9) enable us to estimate the photolithographic resolution in the two cases of non-planarized and planarized PDMS. In this way, we can explain the photolithography experimental observations. In all non-planarized PDMS films the photolithographic resolution is poor apart from at the position of the edge bead where the mask is in good contact with the photoresist layer. In the case of the planarized PDMS films, the photolithographic resolution remains $<5 \mu \mathrm{m}$ up to within 5 $\mathrm{mm}$ of the wafer edge-i.e. $87.3 \%$ of the PDMS surface. When performing photolithography of planarized PDMS film thicknesses $\sim 1000 \mu \mathrm{m}$ obtained using multiple coatings (two here)-the photolithographic resolution is poorer than planarized single coatings but still better than non-planarized PDMS films.

In Figure 10 the red curves (1) correspond to the computed photolithographic resolution on the non-planarized PDMS films - the light blue (2), green (3), and yellow (4) curves correspond to the computed photolithographic resolution obtained when using planarized PDMS films. First, for the nonplanarized films the photolithographic resolution is poor $(\sim 10 \mu \mathrm{m})$ except in the locality of the maximum of the edge bead where the photomask is in contact with the PDMS-see Figures 10(a)-(c). Second, planarization evidently improves the obtainable photolithographic resolution to $<3 \mu \mathrm{m}-$ see blue curves

(2) in Figures 10(a)-(c). In the case of the thickest films (spin speed $=250 \mathrm{rpm}$ ), the photolithographic resolution $<2 \mu \mathrm{m}-$ with the lowest achieved for the planarization period of 72 hours, see the green curve (3) in Figure $10(\mathrm{~d})$. A double coating, used to achieve a thicker film $>1000 \mu \mathrm{m}$, leads to a poorer photolithographic resolution-see yellow curve (4) in Figure 10(d).

\section{Conclusions}


Spin coating polydimethylsiloxane (PDMS) onto silicon wafers at spin speeds between 250-500 rpm results in PDMS film thicknesses in the $200-500 \mu \mathrm{m}$ range. Multiple spin coatings are shown to achieve a total thickness of $\sim 1200 \mu \mathrm{m}$. Rapidly-cured, 'non-planarized' PDMS films have large edge beads which extend $\sim 30 \mathrm{~mm}$ from the wafer edge towards the centre of the wafer. The edge beads are as much as $\sim 150 \mu \mathrm{m}$ thicker than the PDMS film thickness at centre of the wafer. Reflow at room temperature is clearly demonstrated to be an excellent technique for the successful planarization of such spin-coated films-achieving a planarity between $2 \mu \mathrm{m}$ and $15 \mu \mathrm{m}$ depending on spin speed. It is shown that a planarization time of 24 hours is required to achieve good planarity at room temperature. Photolithography has been used to demonstrate the level of planarity of the PDMS films. A photolithographic resolution of $2 \mu \mathrm{m}$ is achieved on planarized spin-coated PDMS films-the photolithographic resolution is $>10 \mu \mathrm{m}$ on non-planarized PDMS films due to poor mask/surface contacting. The study has demonstrated an optimised method for reflow planarization of spin-coated PDMS films and how such films can be used for near-micrometre resolution photolithographic planar processes. In terms of the multiple PDMS coatings, the planarized edge bead remains relatively high, leading to a poorer observed photolithographic resolution. We conclude by saying that if spin-coated thickness $>1000 \mu \mathrm{m}$ are sought, we suggest spin coating in the very low spin speed range of 50-200 rpm where reflow planarization will be enhanced by a thicker viscous film.

\section{Acknowledgements}

The work was in part funded by the French 'Renatech' network. The digital microscope was purchased within the 'TIPTOP_1' project (ANR-16-CE09-0029).

\section{References}


[1] Lötters J C, Olthuis W, Veltink P H and Bergveld P 1997 The mechanical properties of the rubber elastic polymer polydimethylsiloxane for sensor applications J. Micromech. Microeng. 7145

[2] Mata A, Fleischman A J and Roy S 2005 Characterization of polydimethylsiloxane (PDMS) properties for biomedical micro/nanosystems Biomed. Microdevices 7 281-293

[3] Seghir R and Arscott S 2015 Extended PDMS stiffness range for flexible systems Sens. Actuat A 230 33-9

[4] Armani D, Liu C and Aluru N 1999 Re-configurable fluid circuits by PDMS elastomer micromachining Technical Digest. IEEE International MEMS 99 Conference. Twelfth IEEE International Conference on Micro Electro Mechanical Systems (Cat. No.99CH36291) Technical Digest. IEEE International MEMS 99 Conference. Twelfth IEEE International Conference on Micro Electro Mechanical Systems (Cat. No.99CH36291) (Orlando, FL, USA: IEEE) pp 222-7

[5] Unger M A 2000 Monolithic Microfabricated Valves and Pumps by Multilayer Soft Lithography Science 288 113-6

[6] Jo B-H, Van Lerberghe L M, Motsegood K M and Beebe D J 2000 Three-dimensional micro-channel fabrication in polydimethylsiloxane (PDMS) elastomer J. Microelectromechanical Syst. 9 76-81

[7] Shin Y S, Cho K, Lim S H, Chung S, Park S-J, Chung C, Han D-C and Chang J K 2003 PDMS-based micro PCR chip with Parylene coating J. Micromechanics Microengineering 13 768-74

[8] Xia Y, Kim E, Zhao X-M, Rogers J A, Prentiss M and Whitesides G M 1996 Complex Optical Surfaces Formed by Replica Molding Against Elastomeric Masters Science 273 347-9

[9] Xia Y and Whitesides G M 1998 Soft lithography Annu. Rev. Mater. Sci. 28 153-184

[10] Boland J J 2010 Within touch of artificial skin Flexible arrays Nature Matter. 9 790-2

[11] Mannsfeld S C B, Tee B C-K, Stoltenberg R M, Chen C V H-H, Barman S, Muir B V O, Sokolov A N, Reese $C$ and Bao $Z 2010$ Highly sensitive flexible pressure sensors with microstructured rubber dielectric layers Nat. Mater. 9 859-64

[12] Rogers J A, Someya T and Huang Y 2010 Materials and Mechanics for Stretchable Electronics Science 327 1603-7

[13] Wagner S and Bauer S 2012 Materials for stretchable electronics MRS Bull. 37 207-13

[14] Larmagnac A, Eggenberger S, Janossy H and Vörös J 2015 Stretchable electronics based on AgPDMS composites Sci. Rep. 47254

[15] Seghir R and Arscott S 2015 Mechanically robust, electrically stable metal arrays on plasmaoxidized polydimethylsiloxane for stretchable technologies J. Appl. Phys. 118 045309-8

[16] Baëtens T, Pallecchi E, Thomy V and Arscott S 2018 Cracking effects in squashable and stretchable thin metal films on PDMS for flexible microsystems and electronics Sci. Rep. 8 9492-17 
[17] Baëtens, T, Pallecchi E, Thomy V and Arscott S 2019 Metallized SU-8 thin film patterns on stretchable PDMS J. Micromechanics Microengineering 29095009

[18] Cong $\mathrm{H}$ and Pan T 2008 Photopatternable Conductive PDMS Materials for Microfabrication Adv. Funct. Mater. 18 1912-21

[19] Schneider F, Draheim J, Kamberger R and Wallrabe U 2009 Process and material properties of polydimethylsiloxane (PDMS) for Optical MEMS Sens. Actuators Phys. 151 95-9

[20] Koschwanez J H, Carlson R H and Meldrum D R 2009 Thin PDMS Films Using Long Spin Times or Tert-Butyl Alcohol as a Solvent ed M Isalan PLOS ONE 4 e4572

[21] Friend J and Yeo L 2010 Fabrication of microfluidic devices using polydimethylsiloxane Biomicrofluidics 4026502

[22] Long M, Peng S, Deng W, Yang X, Miao K, Wen N, Miao X and Deng W 2017 Robust and thermalhealing superhydrophobic surfaces by spin-coating of polydimethylsiloxane J. Colloid Interface Sci. 508 18-27

[23] Yurgens A 2019 Making thick photoresist SU-8 flat on small substrates J Micromech Microeng 29 017001-5

[24] Stillwagon L E 1987 Planarization of Substrate Topography by Spin Coating J. Electrochem. Soc. 134 2030-7

[25] Bornside D E 1990 Mechanism for the Local Planarization of Microscopically Rough Surfaces by Drying Thin Films of Spin-Coated Polymer/Solvent Solutions J Electrochem Soc 137 2589-95

[26] Stillwagon LE and Larson R G 1990 Leveling of thin films over uneven substrates during spin coating Phys. Fluids Fluid Dyn. 2 1937-44

[27] Bornside D E and Brown R A 1991 Global planarization of spun-on thin films by reflow Appl Phys Lett 581181

[28] Liu H, Herrnsdorf J, Gu E and Dawson M D 2016 Control of edge bulge evolution during photoresist reflow and its application to diamond microlens fabrication J. Vac. Sci. Technol. B 34 021602-6

[29] Kirchner R and Schift H 2019 Thermal reflow of polymers for innovative and smart 3D structures: A review Mater. Sci. Semicond. Process. 92 58-72

[30] Kern W 1990 The Evolution of Silicon Wafer Cleaning Technology J. Electrochem. Soc. 137 1887-92

[31] Oliver J F, Huh C and Mason S G 1977 Resistance to spreading of liquids by sharp edges J. Colloid Interface Sci. 59 568-81

[32] Lawrence C J 1988 The mechanics of spin coating of polymer films Phys. Fluids 31 2786-95

[33] Middleman S 1987 The effect of induced air-flow on the spin coating of viscous liquids J. Appl. Phys. 62 2530-2 
[34] Shu W, Yu X, Hu R, Chen Q, Ma Y and Luo X 2017 Effect of the substrate temperature on the phosphor sedimentation of phosphor-converted LEDs 2017 18th International Conference on Electronic Packaging Technology (ICEPT) 2017 18th International Conference on Electronic Packaging Technology (ICEPT) (Harbin, China: IEEE) pp 398-401

[35] Orchard S E 1963 On surface levelling in viscous liquids and gels Appl. Sci. Res. 11 451-64

[36] Emslie A G, Bonner F T and Peck L G 1958 Flow of a Viscous Liquid on a Rotating Disk J. Appl. Phys. $29858-62$

[37] Kheshgi H S and Scriven L E 1988 The evolution of disturbances in horizontal films Chem. Eng. Sci. $43793-801$

[38] Sötebier C, Michel A and Fresnais J 2012 Polydimethylsiloxane (PDMS) Coating onto Magnetic Nanoparticles Induced by Attractive Electrostatic Interaction Appl. Sci. 2 485-95

[39] Thompson L F, Willson C G and Bowden M J 1994 Introduction to microlithography (Washington, DC: American Chemical Society) 